\section{Genetic Compatibility Affects Queen and Worker Caste Determination}

\author{
Tanja Schwander* ${ }^{\dagger}$ and Laurent Keller
}

$\mathrm{T}$ The mechanisms through which a single genotype can give rise to multiple phenotypes are still unknown (1). Ants provide an example of phenotypic plasticity, in which females develop into two highly differentiated castes: reproductive queens and functionally sterile workers (1). Kin selection theory predicts that the differences between castes stem from environmental effects influencing developmental processes (2). We tested whether phenotypic plasticity could also be affected by interactions between parental genomes. We hypothesized that some genetic combinations are more likely to develop into queens, whereas others are more likely to produce workers. If this is true, genetic effects may be maintained over evolutionary time because the fitness of an allele and whether it is associated with the queen or worker phenotype would depend on its genetic background. Although never investigated, genetic compatibility effects may also greatly constrain the evolution and maintenance of plasticity in other polyphenic species. Accordingly, the ability of a given queen to produce workers and new queens should depend on her own genotype and the genotype of the males with which she mates. We examined caste development in the harvester ant Pogonomyrmex rugosus, which is characterized by colonies comprising a single, multiply mated queen with discrete periods of queen and worker production.

In colonies with a multiply mated queen, genetic compatibility effects on caste determination should translate into a shift in patriline (i.e., offspring fathered by the different mates of the queen) frequencies over the development from eggs to workers versus eggs to queens. Genetic markers were used to infer egg, worker, and queen paternity in each of five colonies founded by naturally mated queens. As predicted, patriline frequencies were similar for eggs laid during the period of queen production and eggs laid during the period of worker production (five independent $G$ tests: $G=$ 0.15 to $0.89 ; \mathrm{df}=4$ to $7 ; P=0.89$ to 0.99 ) but often differed significantly between eggs and workers, eggs and queens, and queens and workers. In each case, significant differences were found in three out of the five colonies (significant differences between new queens and workers found in colonies B20, B22, and B24; $G$ tests $G=12.3$ to 14.3 , $\mathrm{df}=4$ to 7 , and all $P<0.05$; between eggs and workers in colonies B20, B75, and B381; $G$ tests $G=9.5$ to $14.2, \mathrm{df}=5$ to 7 , and all $P<0.05$; and between eggs and queens in colonies B75, B22, and B24; $G$ tests $G=11.9$ to 16.3 , $\mathrm{df}=4$ to 6 , and all $P<0.05$ ).

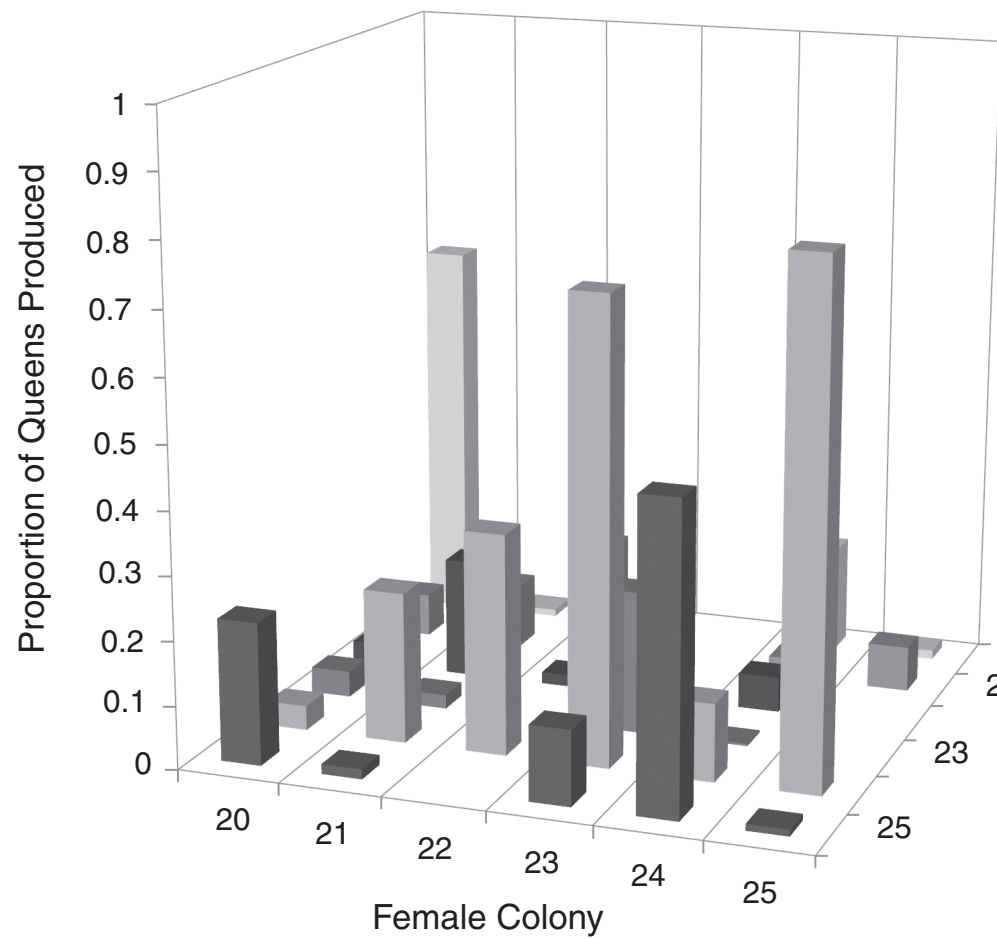

21

Male Colony 1 July 2008; accepted 27 August 2008

Fig. 1. The proportion of new queens produced by singly mated queens in 30 crosses between pairs of parental colonies is shown to be significantly affected by an interaction of male and female colony of origin (each bar depicts the average for all queens per cross; mean number of queens per cross is 6.2 , with a range from 1 to 11 ).
$10.1126 /$ science. 1162590

Department of Ecology and Evolution, University of Lausanne, CH-1015 Lausanne, Switzerland.

*Present address: Department of Biological Sciences, Simon Fraser University, 8888 University Drive, Burnaby, BC V5A 156, Canada.

†To whom correspondence should be addressed. E-mail: tanja.schwander@gmail.com

To directly test for genetic interaction effects on female caste, we conducted controlled crosses in (3). Because reproduction is monopolized from the same colony are genetically more similar than offspring from different colonies. Hence, genetic compatibility effects would result in an interaction between male and female colony of origin on the caste proportion produced by queens from the controlled rosses, whereas genetic predispositions of certain two-way analysis of variance revealed no main effect of either the colony of origin of males $\left(F_{5,19}=0.78, P=\right.$ 0.58) or females $\left(F_{5,19}=0.69, P=0.63\right)$ but a between the parental colonies on the proportion of new queens and workers produced (Fig. 1).

Because genetic compatibility effects strongly interfere with phenotypic plasticity, our results

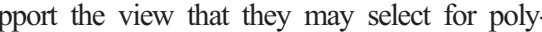
Compatibility effects could evolve as a generate antagonistic coevolution and genotype-bygenotype interactions (5). They are likely to be particularly important in species where selection aries between alternate phenotypes because such in can further generate ontogenetic conflicts likely to be common in social insects and may provide an important step toward the evolution of ng genetic effects on caste and the specialization in the production of specific castes among queens multiple queen colonies [e.g., (7)].

. H. F. Nijhout, Bioscience 49, 181 (1999).

Colonies: Sex Allocation and Kin Selection (Oxford Univ. Press, Oxford, 1996).

. Materials and methods are available as supporting

75, 21 (2000)

W. R. Rice, in Endless Forms. Species and speciation, Oxford, 1998), pp. 261-270

6. A. K. Chippindale, J. R. Gibson, W. R. Rice, Proc. Natl. Acad. Sci. U.S.A. 98, 1671 (2001).

7. R. Kümmerli, L. Keller, Behav. Ecol. 18, 375 (2007). We thank C. Campbell for help in the field; F. Dessimoz and P. Christe, M. Chapuisat, and D. Queller for comments; and K. Parker and D. Arbuthnott for help with English. Supported by the Swiss National Science Foundation.

Supporting Online Material

www.sciencemag.org/cgi/content/full/322/5901/552/DC1 Materials and Methods

Table S1

References

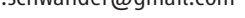

